\title{
RANGE MIGRATION ALGORITHM IN THE PROCESSING CHAIN OF SIGNALS OF A GROUND-BASED SAR SENSOR
}

\author{
B. Hosseiny ${ }^{1,}$ *, J. Amini ${ }^{1}$, M. Esmaeilzade ${ }^{1}$, M. Nekoee ${ }^{1}$ \\ ${ }^{1}$ School of Surveying and Geospatial Engineering, College of Engineering, University of Tehran, Tehran, Iran - (ben.hosseiny, \\ jamini, m.esmaeilzade, mehran.nekoee) @ut.ac.ir
}

Commission III, WG III/2

KEY WORDS: Ground-Based System, Radar Imaging, Synthetic Aperture Radar, S-band, System Development, Image Formation, Radar Signal Processing, Range Migration

\begin{abstract}
:
Synthetic aperture radar (SAR) system based on frequency modulated continuous wave (FM-CW) transmission is a viable option for producing high-resolution ground-based imaging radars. Compared with pulsed SAR systems, the combination of FM-CW technology and SAR processing techniques have the advantages of small cubage, lightweight, cost-effectiveness, and high resolution in the SAR image. These characteristics make FM-CW SAR suitable to be deployed as payload on ground Based SARs (GB-SARs) for environmental and civilian applications. In this paper, the Range Migration Algorithm (RMA) is used in the processing chain of a Ground-Based SAR (GB-SAR) sensor. The mentioned sensor has been developed in Microwave Remote Sensing Laboratory (MReSL) at the School of Surveying and Geospatial Engineering, the University of Tehran for the generation of a complex image from the raw signal. The raw signal is acquired with that sensor working at S-band, frequency modulating from $2.26 \mathrm{GHz}$ to 2.59 GHz.
\end{abstract}

\section{INTRODUCTION}

In recent years, there has been a growing interest in frequency modulated continuous wave (FM-CW) SAR technology for various military and civilian applications. Compared with pulsed SAR, the combination of FM-CW technology and SAR processing techniques have the advantages of small cubage, lightweight, cost-effectiveness, and high resolution in the SAR image. These characteristics make FM-CW SAR suitable to be deployed as payload on Ground-Based SARs (GB-SARs) for environmental and civilian applications.

Researchers have investigated several FMCW-SAR architectures: (Charvat and Kempel, 2006) Developed an ultralow-cost FMCW GB-SAR imaging system, operating on an automatic rail. (Charvat et al., 2010) developed an ultrawideband (UWB) rail-SAR system with $2 \mathrm{GHz}$ of bandwidth (1.926-4.069 GHz chirp in $10 \mathrm{~ms}$ ) for through-wall detection purposes. This system was capable of imaging targets in shape of a cylinder with $15 \mathrm{~cm}$ in height and $1 \mathrm{~cm}$ in diameter behind a $10 \mathrm{~cm}$ thick lossy dielectric slab. (Sanz-Marcos et al., 2007) developed a bistatic ground-based SAR system, which receives transmitted radar signal of the airborne or satellite platform. A tomographic ground-based SAR system was developed in (Ji et al., 2014) for 3-D SAR imaging.

Many signal processing algorithms have been developed to generate a focused image of the SAR signal. These algorithms are divided into two major groups: (1) Time-domain algorithms: such as Backprojection (BP) and Time-Domain Correlation (TDC) algorithms and (2) Frequency-domain algorithms: such as, Range Stacking algorithm (RSA), Polar Format Algorithm (PFA), Frequency scaling algorithm (FSA), Range Doppler algorithm (RDA), range migration algorithm (RMA), and Chirp Scaling algorithm (CSA). (Okoń-Fąfara et al., 2016; Yigit et al., 2013) compared the performance of time-domain and frequency-domain algorithms in FMCW SAR systems. (Reigber et al., 2013) Reviewed signal processing and imaging algorithms for very high-resolution SAR systems including time-domain algorithms, frequency-domain algorithms, and error correction methods. (Charvat and Kempel, 2006) Implemented four imaging algorithms in frequency-domain (PFA, simplified PFA, RMA, and RSA) to create a focused image of FMCW rail-SAR system.

In this paper, Range Migration Algorithm (RMA) is used in the processing chain of a GB-SAR sensor, which has been developed in the Microwave Remote Sensing Laboratory (MReSL) at the School of Surveying and Geospatial Engineering, University of Tehran, Tehran, Iran for the generation of a complex image from raw signal. We present the analytical process of the Frequency Modulation Continuous Wave (FMCW) SAR signals at S-band for generating a complex image. This paper is organized into sections as follows. In Section 2, the methodology will be presented. Range Migration Algorithm in the GB-SAR signal processing chain will be addressed in section 3. Experimental results demonstrate and discuss in section 4 . And finally, we will conclude this paper in section 5 .

\section{METHODOLOGY}

In this section, we are going to describe required methods in processing of received radar signals in order to calculate a target's range and create high-resolution radar image by synthetic aperture processing (SAR-imaging).

\subsection{Frequency-Modulated (FM) Signal}

In frequency-modulated technology, radar transmits a signal with varying frequency as a function of time. This kind of signal is also known as chirp signal. Various modulations are possible for signal, but linear modulation is the most common approach. 
In linear frequency modulation (LFM), the frequency varies linearly as a function of time (Figure 1) (Li et al., 2017).

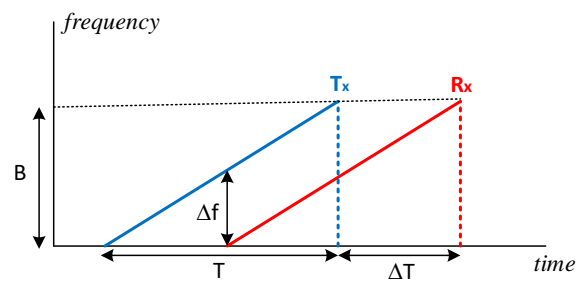

Figure 1. Linear Frequency modulated signal

Figure 2 shows the block diagram of a Frequency Modulated Continuous Wave (FMCW) radar system. The transmitted signal in linear frequency modulated radar, for one sweep duration $(\mathrm{T})$, can be expressed as:

$$
s_{t}=\exp \left\{j 2 \pi f_{c} t+j \pi c_{r} t^{2}\right\}
$$

where $\quad f_{c}=$ carrier frequency

$$
\begin{aligned}
c_{r} & =\mathrm{B} / \mathrm{T} \text { chirp rate } \\
t & \in[0, T]
\end{aligned}
$$

The received signal is the delayed version of the transmitted signal:

$$
s_{r}=\exp \left\{j 2 \pi f_{c}\left(t-t_{d}\right)+j \pi c_{r}\left(t-t_{d}\right)^{2}\right\}
$$

where $\quad t_{d}=$ time delay

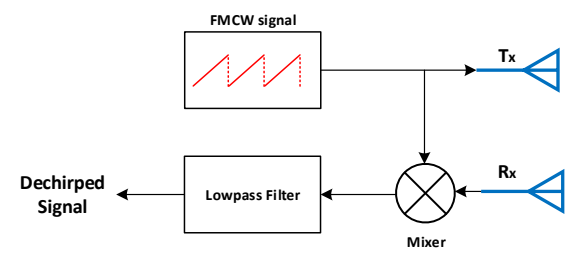

Figure 2. LFMCW radar system

Dechirping process is needed to measure the range. Dechirping is done by mixing a replica of the transmitted signal with the received signal and lowpass filtering. Therefore, the beat signal (in some literature intermediate signal) is generated (Meta et al., 2007):

$$
s_{b}=\exp \left\{j 2 \pi f_{c} t_{d}+j 2 \pi c_{r} t_{d} t-j \pi c_{r} t_{d}{ }^{2}\right\}
$$

Target's range can be measured from the beat frequency, which is proportional to the time delay $\left(f_{b}=c_{r} t_{d}\right)$ :

$$
R=\frac{c f_{b} T}{2 B}
$$

Equation (4) expresses the direct relation between the beat frequency echo range. Thus, we can calculate range by Fourier transform acquired beat signal.

Range resolution in FM radar is proportional to transmitted signal's bandwidth:

$$
\delta R=\frac{c}{2 B}
$$

This equation means that we can obtain better resolution by larger modulation bandwidth, which is one of the most important properties of FM signals.

\subsection{Synthetic Aperture Radar}

The main idea of synthetic aperture radar imaging is to increase radar resolution in azimuth direction by coherent integration of received signals in different azimuth position of the radar sensor. Figure 3 shows the imaging geometry of a GB-SAR that operates on a linear rail. In this system, the radar sensor is mounted on a rail and in every azimuth step, acquires data in a range profile (Charvat, 2014).

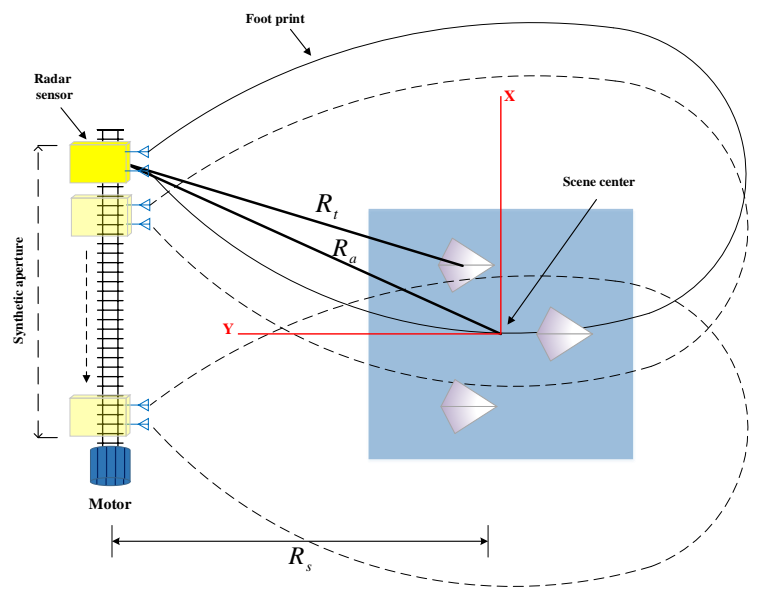

Figure 3. Rail-SAR imaging system

The corresponding dechirped SAR signal phase, based on Figure 3. is (Carrara et al., 1995):

$$
\begin{aligned}
& \Phi(n, t)= \\
& -\frac{4 \pi}{c}\left[f_{c}+c_{r}\left(t-\frac{2 R_{s}}{c}\right)\right]\left(R_{t}-R_{s}\right)+\frac{4 \pi c_{r}}{c^{2}}\left(R_{t}-R_{s}\right)^{2}
\end{aligned}
$$

where $\quad R_{s}=$ Range from scene-center to flight path

$R_{t}=$ Range from target to sensor at every step

\section{SAR IMAGE FORMATION AND RANGE MIGRATION ALGORITHM (RMA)}

The dechirped signal is used as an input of image formation algorithms for generating the focused SAR image of the scene of interest. Here we use Range migration algorithm (RMA) (Cafforio et al., 1991) in order to generate SAR image from the raw signal. RMA operates in the frequency domain. Therefore, it is computationally efficient in comparison with time-domain algorithms such as Backprojection (Carrara et al., 1995; Reigber et al., 2013). Figure 4 shows the block diagram of the RMA steps. RMA consists of four main steps (Carrara et al., 1995):

1) Along-track Fourier transform: One dimensional Fourier transform maps SAR signal data into wavenumber space $\left(K_{X}, K_{R}\right)$, where $K_{X}$ is azimuth spatial frequency varying linearly from $-\frac{\pi}{\Delta x}$ to $\frac{\pi}{\Delta x}$ where $\Delta x$ is the interpulse sample spacing in units of 
distance and $K_{R}$ is range spatial frequency varying linearly from $\frac{4 \pi f_{c}}{c}$ to $\frac{4 \pi\left(f_{c}+B\right)}{c}$.

2) Matched Filtering: The resulting data is chirped in $K_{X}$ dimension. The matched filter removes the chirp in the azimuth frequency domain. Additionally, implementing a matched filter perfectly corrects the range curvature of scatterers at the same range as scene center $\left(R_{s}\right)$ and partially compensates range curvature of scatterers at other ranges. Matched filter equation is:

$\mathrm{H}_{m f}\left(K_{X}, K_{R}\right)=$

$\exp \left\{-j\left(R_{S} K_{R}\right)+j\left(R_{S} \sqrt{K_{R}{ }^{2}-K_{X}{ }^{2}}\right)\right\}$

3) Stolt interpolation: Stolt interpolation is onedimensional transformation mapping $K_{R}$ into $K_{Y}$ domain, where $K_{Y}$ is equal to $\sqrt{K_{R}{ }^{2}-K_{X}{ }^{2}}$. It removes residual range curvature of all scatterers.

4) 2-D inverse Fourier transform: The last step is performed to return data to the time domain. By computing two-dimensional inverse Fourier transform final focused image appears in range and azimuth dimensions.

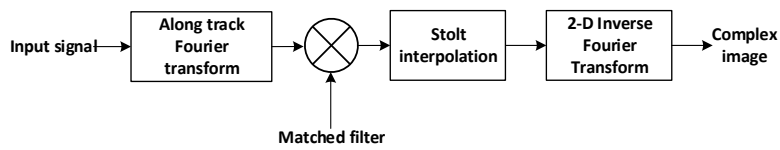

Figure 4. Range Migration Algorithm block diagram

\section{EXPERIMENTAL RESULTS}

In this section, we demonstrate our experimental tests and results we had on our developed ground-based SAR system. Table 1 shows the main characteristics of this system.

\begin{tabular}{ll}
\hline Signal Type & FMCW \\
Carrier frequency $\left(\mathrm{f}_{\mathrm{c}}\right)$ & $\begin{array}{l}2.425 \mathrm{GHz} \\
(\mathrm{S}-\mathrm{band})\end{array}$ \\
$\begin{array}{l}\text { Bandwidth }(\mathrm{B}) \\
\text { Sweep time }(\mathrm{T})\end{array}$ & $20 \mathrm{MHz}$ \\
$\begin{array}{l}\text { Radar steps in the azimuth } \\
\text { direction }\end{array}$ & $1 \mathrm{~cm}$ \\
$\begin{array}{l}\text { Aperture length } \\
\text { range resolution }\end{array}$ & $140 \mathrm{~cm}$ \\
& $\frac{c}{2 B}=45 \mathrm{~cm}$ \\
\hline
\end{tabular}

Table 1. GB-SAR main characteristics

\subsection{Tests on Radar Ranging}

Before SAR imaging experiments, the range processing of radar system is done by moving a target in front of radar antennas. As we mentioned in the previous section, the target's range can be determined by Fourier transform of the received dechirped signal. Figure 5 compares acquired signals at different times based on target moving behavior. Figure 5(a) shows the raw SAR signal. We can see that when the target is close to the radar sensor, received echo is strong and contains lower frequency. As the target farther away, the power of the echo decreases and frequency increases. Part (b) demonstrates raw signals of part (a) after Fourier transform that contains the target's range information.

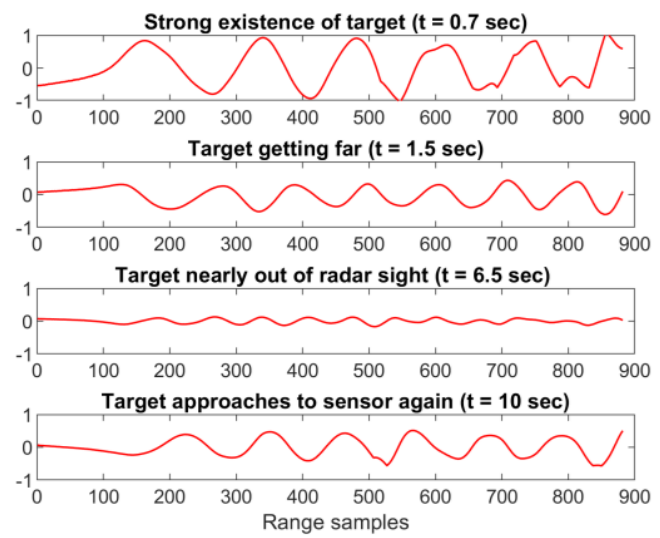

(a)
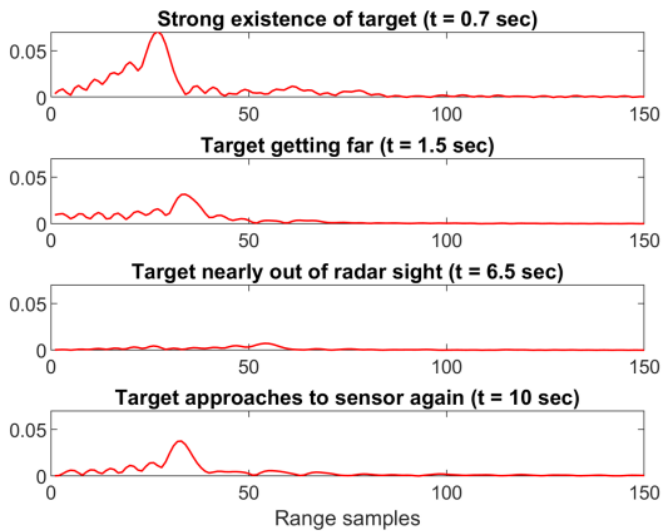

(b)

Figure 5. Examples of (a) raw signal (b) compressed range signal in radar ranging experiment

Figure 6 shows the full range-time image (RTI) of experimented scenario. $\mathrm{X}$-axis expresses range in meter and $\mathrm{Y}$-axis expresses time in second. Static targets are appeared as straight lines and moving target is appeared as inclined lines: in the top left corner of the figure target is getting away of radar and then it approaches to the transmitter during 25 seconds and again this approach is repeated one more time until $t$ equals to 50 seconds.

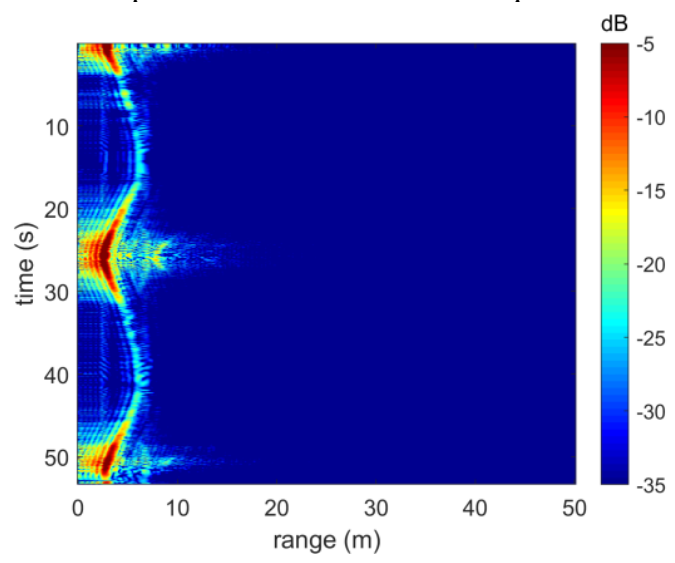

Figure 6. Range-Time image of moving target in front of the radar sensor 
In order to subtract static targets as clutters, we used two pulses canceling approach by subtracting pulse at time $t_{i-1}$ from pulse at time $t_{i}$. Based on this approach, resolution bins containing static targets have the same values and they are canceled by subtraction of two pulses. Figure 7 shows clutter rejected range time image.

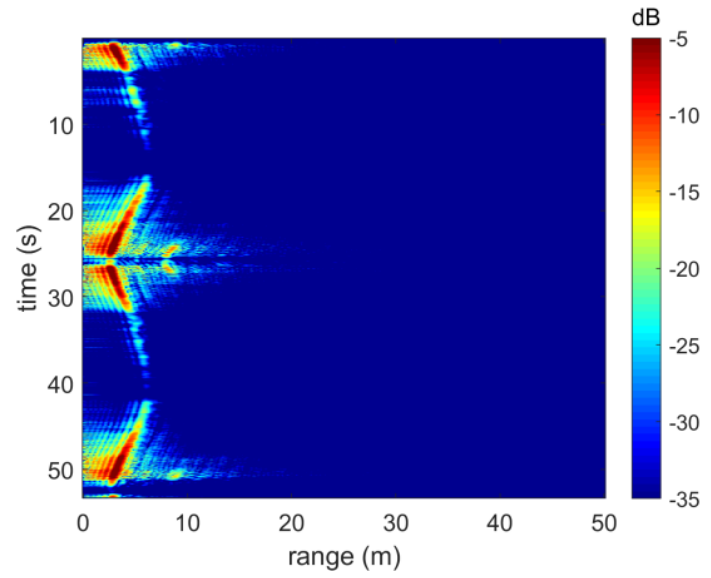

Figure 7. Range-Time image of moving target in front of the radar sensor after removing static targets as clutter

\subsection{Synthetic Aperture Radar Imaging Results}

Our final test is on SAR imaging capability of the developed sensor. According to table 1 sensor travels 140 steps with $1 \mathrm{~cm}$ intervals, and in every step transmits and receives signals. Figure 9 shows SAR imaging result of a scene including one scattering target. As it is evident from Figure 9 (a) backscattered signals from the scene are completely distributed in range and azimuth direction. Therefore, targets cannot be distinguished in raw 2-D signal of SAR imaging. After processing and range and azimuth compression, focused target can be distinguished from the background (Figure $9(\mathrm{~b})$ ).

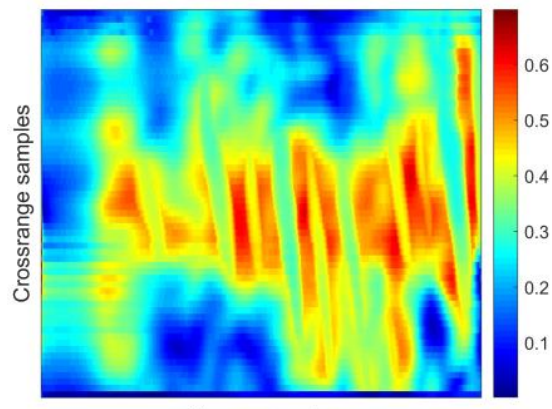

(a)

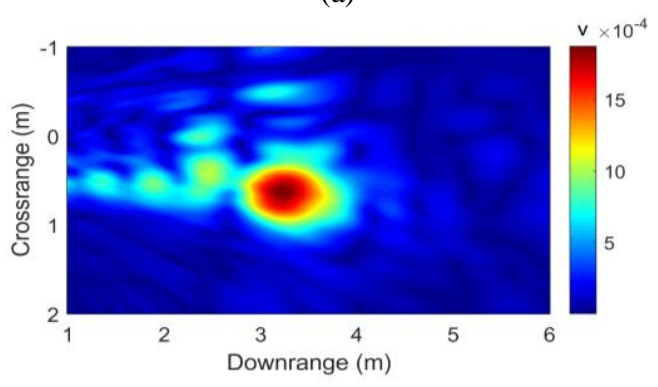

(b)

Figure 9. SAR imaging result. (a) raw 2-D signals (b) focused final SAR image
With another perspective view in $3 \mathrm{D}$, Figure 10 shows the steps of the signal processing chain in $3 \mathrm{~d}$ for more details. The first and second dimensions are referred to the range and azimuth directions and the third dimension indicates the absolute value of signals. It is important to note that in ground-based SAR scenario, $R_{s}$ is considered to be zero. Therefore, the matched filtering step automatically is skipped.

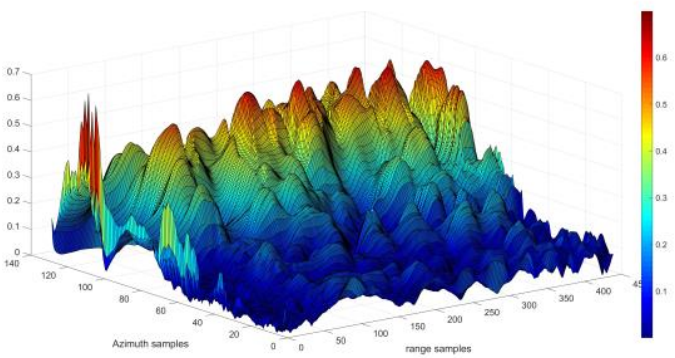

(a)

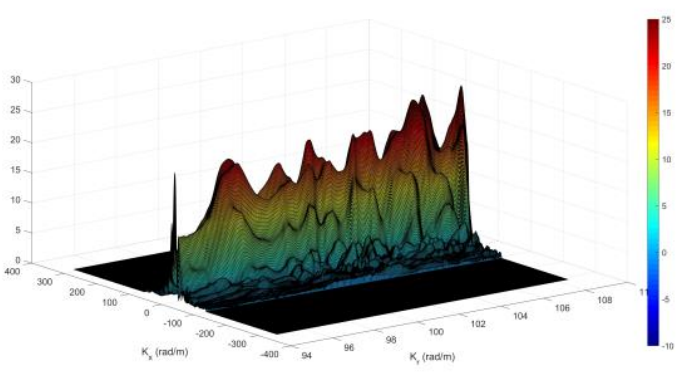

(b)

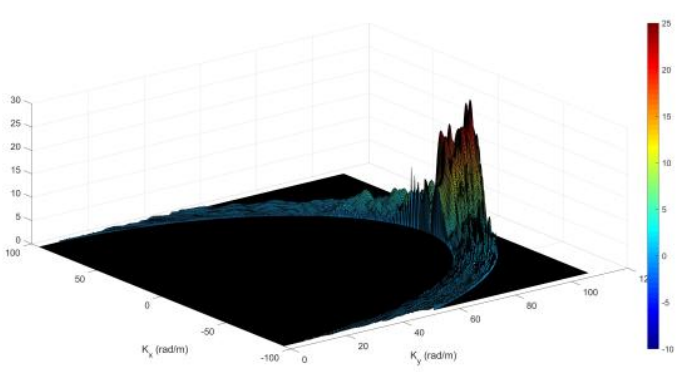

(c)

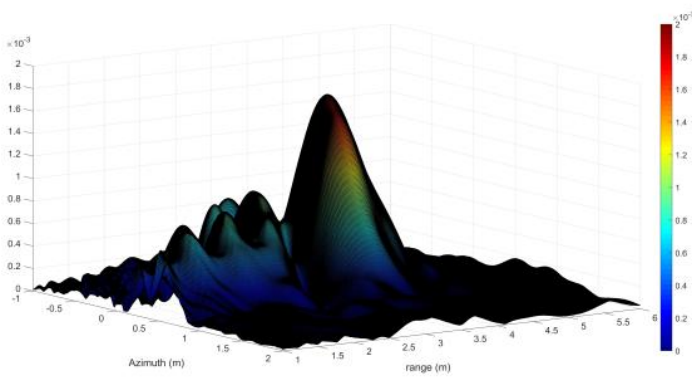

(d)

Figure 10. RMA steps in SAR image generation. (a) raw 2-D signals as algorithm's input (b) signals after along-track Fourier transform and initial phase compensations (c) signals after Stolt interpolation (d) signals after two-dimensional inverse Fourier transform (focused signal)

Focusing capability of the generated SAR image is evaluated with the peak sidelobe ratio (PSLR) of the focused target in the range and azimuth direction of the imaging scene. PSLR is the 
difference between the first sidelobe and the peak value of the signal in decibels. The obtained PSLR of the focused target in the range and azimuth directions are -5.85 and $-13.69 \mathrm{~dB}$, respectively.

\section{CONCLUSION}

This paper explained processing steps required to generate highresolution synthetic aperture radar image from acquired raw signals of a ground-based FMCW radar system. The frequencydomain Range migration algorithm was used as the SAR image formation algorithm. In the end, we presented two experiments included moving target detection in radar mode and synthetic aperture radar imaging of a scattering target.

\section{REFERENCES}

Cafforio, C., Prati, C., Rocca, F., 1991. SAR data focusing using seismic migration techniques. IEEE Trans. Aerosp. Electron. Syst. 27, 194-207. https://doi.org/10.1109/7.78293

Carrara, W.G., Goodman, R.S., Majewski, R.M., 1995. Spotlight synthetic aperture radar : signal processing algorithms. Artech House, Boston :

Charvat, G.L., 2014. Small and short-range radar systems. CRC Press.

Charvat, G.L., Kempel, L.C., 2006. Synthetic aperture radar imaging using a unique approach to frequency-modulated continuous-wave radar design. IEEE Antennas Propag. Mag. 48, 171-177. https://doi.org/10.1109/MAP.2006.1645606

Charvat, G.L., Kempel, L.C., Rothwell, E.J., Coleman, C.M., Mokole, E.L., 2010. A through-dielectric radar imaging system. IEEE Trans. Antennas Propag. 58, 2594-2603.

Ji, Y., Han, H., Lee, H., 2014. Construction and application of tomographic SAR system based on GB-SAR system, in: Geoscience and Remote Sensing Symposium (IGARSS), 2014 IEEE International. IEEE, pp. 1891-1894.

Li, C., Peng, Z., Huang, T.-Y., Fan, T., Wang, F.-K., Horng, T.S., Muñoz-Ferreras, J.-M., Gómez-García, R., Ran, L., Lin, J., 2017. A review on recent progress of portable short-range noncontact microwave radar systems. IEEE Trans. Microw. Theory Tech. 65, 1692-1706.

Meta, A., Hoogeboom, P., Ligthart, L.P., 2007. Signal processing for FMCW SAR. IEEE Trans. Geosci. Remote Sens. $45,3519-3532$.

Okoń-Fąfara, M., Serafin, P., Kawalec, A., 2016. An analysis of Chosen Image Formation Algorithms for Synthetic Aperture Radar with FMCW. Int. J. Electron. Telecommun. 62, 323-328. https://doi.org/10.1515/eletel-2016-0044

Reigber, A., Scheiber, R., Jager, M., Prats-Iraola, P., Hajnsek, I., Jagdhuber, T., Papathanassiou, K.P., Nannini, M., Aguilera, E., Baumgartner, S., Horn, R., Nottensteiner, A., Moreira, A., 2013. Very-High-Resolution Airborne Synthetic Aperture Radar Imaging: Signal Processing and Applications. Proc. IEEE 101, 759-783. https://doi.org/10.1109/JPROC.2012.2220511
Sanz-Marcos, J., Lopez-Dekker, P., Mallorqui, J.J., Aguasca, A., Prats, P., 2007. SABRINA: A SAR bistatic receiver for interferometric applications. IEEE Geosci. Remote Sens. Lett. 4, 307-311.

Yigit, E., Demirci, S., Ozdemir, C., Tekbas, M., 2013. Shortrange ground-based synthetic aperture radar imaging: performance comparison between frequency-wavenumber migration and back-projection algorithms. J. Appl. Remote Sens. 7, 73483 . 